EDITORIAL

\title{
INGENIARE: REVISTA CHILENA DE INGENIERÍA
}

Las revistas científicas constituyen el instrumento imprescindible para impulsar la difusión social del conocimiento científico y tecnológico. El conocimiento, como acervo de la humanidad, aumenta y se enriquece cuando quienes lo generan publican el resultado de sus investigaciones como informes o artículos científicos. Así, para dar a conocer los resultados alcanzados por la investigación que se desarrolla en los países de la región, es necesario identificar, estimular y apoyar aquellas revistas que en cada país se destacan por la calidad de sus contenidos y así fortalecer el proceso de difusión de conocimientos generados en la región.

Dentro de este contexto, y dado que el objetivo de la comunicación científica es el registro, evaluación, diseminación y acumulación del conocimiento, para contribuir al avance del conocimiento, el investigador -en este caso el investigador de la región-asumirá este compromiso utilizando los medios adecuados, lo que será posible en la medida en que el artículo sea publicado en una revista que resulte visible y accesible para la comunidad científica.

En el último tiempo se han producido cambios revolucionarios en la forma de comunicación del conocimiento y en la organización de la ciencia, pasándose de un sistema organizado alrededor de una disciplina a un sistema organizado alrededor de problemas transdisciplinarios. En este nuevo paradigma destacan las relaciones interpersonales y emerge la interacción entre grupos e instituciones de investigación, que influyen en el proceso de comunicación y de producción científica y tecnológica, contribuyendo, al mismo tiempo, al desarrollo global.

A partir de la literatura sobre investigación y difusión científica de reciente data, los investigadores, tomando en consideración que están sujetos a la evaluación por pares, en muchas instancias demuestran cautela en la selección de revistas a las cuales someterán los manuscritos para su publicación, lo que es comprensible, ya que parte del éxito del autor depende de factores como la habilidad y competencia del editor de la revista, de la política editorial de la publicación, de la presentación del artículo y de las normas recomendadas por los organismos internacionales que regulan los estándares que rigen las publicaciones.

Por otro lado, no es difícil comprender la posición de los editores que deben pensar en la revista científica no solamente como un vehículo para perpetuar el conocimiento, sino que, debido a las grandes transformaciones y la alta velocidad de los procesos de comunicación, como impulsora del proceso de generación y difusión de la información, mediante el acceso a las nuevas bibliotecas digitales y virtuales, donde se encuentran las publicaciones de mayor impacto.

Uno de los aspectos preocupantes de las nuevas condiciones de la difusión del conocimiento científico es la ausencia de una capacitación formal que respalde la labor de los editores, y les otorgue un carácter profesional, condición en la que Chile no constituye excepción, dado que la tarea es enfrentada con mucho entusiasmo pero con pocos conocimientos al respecto.

Otro aspecto que inquieta al investigador es la desigualdad de criterios respecto de las normas de publicación y evaluación de los artículos, que en general son realizadas solo por pares, quienes aceptan o rechazan el nuevo conocimiento, ignorando criterios como la competitividad, el costo y la aceptación por parte de la sociedad científica que -en última instancia- es la que juzga y en definitiva acepta o rechaza el nuevo conocimiento.

En cada campo hay revistas de prestigio que reciben más manuscritos, tienen estándares más rigurosos e índices de rechazo más altos. La evaluación de las revistas científicas, como todos los procesos que afectan a los proyectos de investigación, a la adjudicación de ayudas para investigación y a todo lo involucrado en la temática, constituyen en general procesos complejos poco debatidos, cuyos criterios son: 
- Detectar las características que determinan las formalidades y calidad científica de la revista.

- Mejorar la calidad de las revistas como medio de comunicación científica e instrumento de evaluación de la actividad científica.

- Incrementar el nivel de prestigio y reconocimiento nacional, regional e internacional de la revista.

- Medir el impacto social del nuevo conocimiento generado, utilizando las nuevas alternativas de acceso vigentes.

\section{Ingeniare - Revista Chilena de Ingeniería}

Al evaluar nuestra revista lo hacemos según criterios y normas preestablecidos tendientes a homogeneizar la presentación y preservar e incrementar su calidad. Para evaluar esta calidad se utilizan una serie de parámetros como:

1. El factor de impacto, parámetro del Journal Citation Reports del Institute for Scientific Information (ISI) que consiste en un índice que expresa la relación entre el número de citas recibidas por una revista en un año determinado y el número de artículos que esta revista publicó en los dos años anteriores.

2. Notoriedad de los colaboradores en cada campo, que en el caso de nuestra revista consiste en colaboraciones de importantes autores nacionales y extranjeros.

3. La estabilidad de la revista, que en nuestro caso tiene 12 años de difusión continua, comenzando con un volumen, de un número al año, para culminar con tres números anuales a partir de 2005.

4. El renombre de la institución que patrocina la revista. En nuestro caso es editada por la Universidad de Tarapacá, institución estatal perteneciente al Consejo de Rectores de Universidades Chilenas, que es una universidad regional de prestigio en el área de la ingeniería.

5. El idioma de la publicación, que en el caso de Ingeniare son el español, inglés y portugués, con traducción a inglés de los resúmenes y la editorial.

6. Las normas nacionales e internacionales de publicación, que, como revista indexada, son aquellas indicadas por la Scientific Electronic Library Online, SciELO.

7. El Comité Editor y cuerpo de evaluadores externos, principales filtros de calidad de la revista. En Ingeniare están conformados por destacados académicos de universidades e instituciones chilenas y extranjeras como: la Universidade Estadual de Campinas-Brasil, Universidad de Santiago de Chile, Universidad Católica de Chile, Universidade de Brasilia, Universidad Politécnica de Madrid, Universidad Federico Santa María, Universidad de Tarapacá y el European Journal of Engineering Education.

La evaluación de revistas de difusión científica y académica tiene como objetivo primordial el homogeneizar la presentación e incrementar la calidad de los contenidos, a la vez de incrementar el nivel de prestigio y reconocimiento de la publicación.

En nuestro caso, Ingeniare, como revista emergente, tiene la oportunidad de innovar en el campo de la comunicación científica, aplicando para ello los parámetros cuantitativos y cualitativos de revistas de gran prestigio. Es lo que pretendemos hacer en los años venideros, para alcanzar reconocimiento a nivel regional e internacional.

Carlos Villarroel González

Editor

Ingeniare - Revista Chilena de Ingeniería

Arica, Chile 